\title{
Biocompatibility of Human Whartons Jelly Mesenchymal Stem Cells on Poly-caprolactone and Collagen Based Nanofiber Mats
}

\author{
E. Lafond, A. Lawson, R. Niemeier, C. Cady, K. Nair \\ Bradley University \\ 1501 W. Bradley Avenue \\ Peoria, IL 61625
}

\begin{abstract}
Nanofiber scaffolds were fabricated to analyze the proliferation of human Whartons Jelly Mesenchymal Stem Cells (hWJMSCs) for skin tissue engineering applications. Polycaprolactone (PCL) and PCL mixed with collagen scaffolds were fabricated using electrospinning. ImageJ analysis was carried out on SEM images to characterize the structural and morphological properties of the nanofiber scaffolds. Tensile testing was also performed to quantify the mechanical properties of the scaffolds. The scaffolds were then seeded with hWJMSCs to study cell proliferation over five days in order to determine the feasibility for tissue regeneration. The average fiber diameters for the PCL scaffold and for the PCL/collagen scaffold were $0.542 \mu \mathrm{m}$ and $0.633 \mu \mathrm{m}$, respectively. The Young's Moduli of the PCL and PCL/collagen scaffolds were $0.00370 \mathrm{~Pa}$ and 0.00683 Pa. respectively. After analyzing the data, it can be concluded that the PCL/collagen scaffold is better suited for regeneration of damaged or diseased nervous tissue.
\end{abstract}

\section{INTRODUCTION}

The process of electrospinning is used to create scaffolds made of nanofibers of approximately the same diameter. Scaffolds are of interest for tissue engineering because of their ability to pose as extra-cellular matrices while the tissue is growing and to then biodegrade when placed in the human body, lessening the risk of rejection [1].

PCL is a synthetic polymer that has been used extensively to create scaffolds for tissue engineering [1]. It is used in cartilage, bone, ligament/tendon, and vascular tissue engineering applications because of its ability to increase cell proliferation [1].

Collagen is a natural polymer that has also been used for tissue engineering applications because it resembles the extracellular matrix that surrounds all cells in the human body [1]. When electrospun at certain parameters, collagen has the same properties as that of extra-cellular matrix. When collagen is combined with PCL, it has been shown that cell proliferation is higher for dermal cells $[1,2]$. However, none of the studies have quantified the effects of the structural and mechanical properties of the nanofiber scaffold on hWJMSCs, a cell type ideal for tissue regeneration.

The objectives of this study include analyses of the structural and morphological properties of the scaffolds. This study also quantifies the proliferation of hWJMSCs on the PCL and the PCL/collagen scaffolds over five days.
II. Methods

\section{Solution}

Two types of scaffolds were fabricated-one using PCL alone, and the other using both PCL and collagen. The solution was made using one part methanol, three parts chloroform, and $7.5 \%$ weight per volume of PCL. Collagen was added to the solution to create a final concentration of 5 $\mathrm{mg} / \mathrm{mL}$ (Sigma Aldrich, St. Louis, MO).

\section{Scaffolds}

Scaffolds were created by electrospinning using $5 \mathrm{~mL}$ syringes (BD Rx Inc., Franklin Lakes, NJ) and 27 gauge needles (BD Rx Inc., Franklin Lakes, NJ). To produce both the PCL and the PCL/collagen scaffolds, a voltage of $13 \mathrm{kV}$ was applied using a power supply (Gamma High Voltage Research, Ormond Beach, FL, USA). The flow rate was controlled using a syringe pump (kd Scientific, Holliston, MA, USA) at $3 \mathrm{~mL} / \mathrm{hr}$ for the PCL scaffold, and at $900 \mu \mathrm{L} / \mathrm{hr}$ for the PCL/collagen scaffold. Both scaffolds required a collecting distance of $13 \mathrm{~cm}$, and each were electrospun for thirty minutes [2].

\section{SEM Imaging}

SEM imaging was conducted on both PCL and $\mathrm{PCL} /$ collagen scaffolds. Imaging was done at the Imaging Center, SIU School of Medicine, Springfield, IL. The fiber diameters were quantified using ImageJ software. SPSS was used to determine if there was a significant difference between PCL and PCL/collagen scaffolds.

\section{Tensile Testing}

Specimens were cut out to ASTM standard D-638-V. Specimen thickness was measured at three different locations using a micrometer (Model No. 49e63, Testing Machines Inc., Amityville, NY), and the average thickness was used for calculations. Tensile testing of the scaffolds was conducted using an Instron Universal Testing Machine Model 4201 (Norwood, MA). Physical properties were determined with a crosshead speed of $50 \mathrm{~mm} / \mathrm{min}$, a gauge length of $7.62 \mathrm{~mm}$, and a $1 \mathrm{kN}$ load cell. Stress-strain curves were plotted to find the Young's Moduli. SPSS was used to determine if there was a significant difference between PCL and PCL/collagen Young's Moduli. 


\section{Cell Culture}

Cells were cultured at $5 \% \mathrm{CO}_{2}$, ambient $\mathrm{O}_{2}$, and $37^{\circ} \mathrm{C}$ until $60 \%$ confluency was achieved in a cell culture flask. Cells were then rinsed with PBS and lifted with TrypLE. Cells were centrifuged at $750 \mathrm{xg}$ for eight minutes to pellet cells and remove TrypLE from suspension. Cells were counted, and 5,000 cells were plated onto the nanofibers for one hour. Nanofibers were mounted onto silicone disks and adhered to the bottom of a 24 -well plate using silicone grease. Following plating, $1 \mathrm{~mL}$ of Stem Life cell media was added to each well, and cells were incubated until the appropriate time point for proliferative assessment. Proliferation was assessed by MTS assay. Fiber disks were moved to new wells of a plate and $300 \mu \mathrm{L}$ of MTS (diluted 1:5 with PBS) and incubated for 3-4 hours at $5 \% \mathrm{CO}_{2}$, ambient $\mathrm{O}_{2}$, and $37^{\circ} \mathrm{C}$. Reacted MTS was aliquoted into a 96 -well plate and read at $490 \mathrm{~nm}$ to determine optical density.

\section{Results}

\section{SEM Imaging}

SEM images in Fig. 1 were used to quantify the fiber diameter of both the PCL and the PCL/collagen scaffolds.

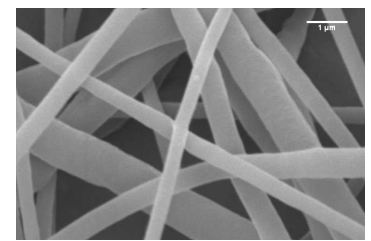

(a)

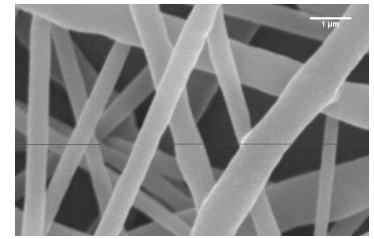

(b)
Fig. 1. SEM images of (a) PCL at 15,000x magnification and (b) $\mathrm{PCL} /$ collagen at $15,000 \mathrm{x}$ magnification.

The average fiber diameter of the PCL scaffold is $0.555 \pm$ $0.076 \mu \mathrm{m}$. The average fiber diameter of the PCL/collagen scaffold is $0.632 \pm 0.090 \mu \mathrm{m}$. An independent student t-test determined that the difference in fiber diameter measurements was not significant $(\mathrm{p}=0.522)$.

\section{Tensile Testing}

Tensile testing was used to quantify the Young's Moduli of the two scaffolds. The PCL scaffold was found to have an average Young's Modulus of $0.00370 \pm 0.0006 \mathrm{~Pa}$, whereas the average Young's Modulus of the PCL/collagen scaffold was $0.00683 \pm 0.0009 \mathrm{~Pa}$. An independent student t-test determined that the difference was significant $(\mathrm{p}=0.048)$. Fig. 2 displays the Young's Modulus for the two samples.

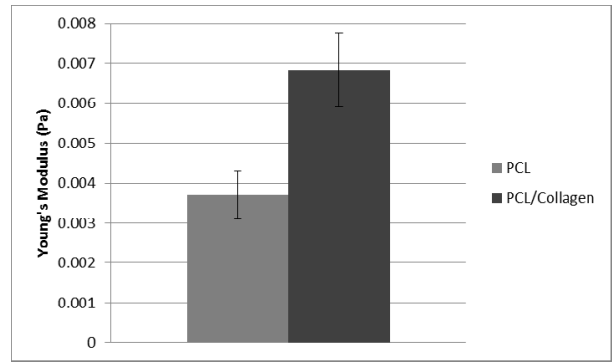

Fig. 2. Young's Moduli of PCL (light gray) and PCL/collagen (dark gray) scaffolds and their corresponding standard errors.

\section{Cell Culture}

Culture of hWJMSCs over a period of five days demonstrated significant proliferation increases on PCL/collagen material over PCL material and a control of cells in a well-plate alone at the early time points of one and two days in vitro (DIV). Significantly increased proliferation on PCL/collagen material was observed as early as DIV2 (one-way ANOVA with a Newman-Keuls post-test, $\mathrm{p} \leq 0.05$ ). These data suggest that inclusion of collagen to the material allows cells to adhere more easily to the nanofiber material and recover more quickly. Faster recovery leads to quicker cell proliferation and overall better cell health. There was no significant difference between groups at DIV3. By DIV5, the PCL material showed significantly increased cell density compared to other groups. These data are summarized in Fig. 3. The decrease in cellular density at DIV5 in the $\mathrm{PCL} /$ collagen material was likely due to contact inhibition of the cells.

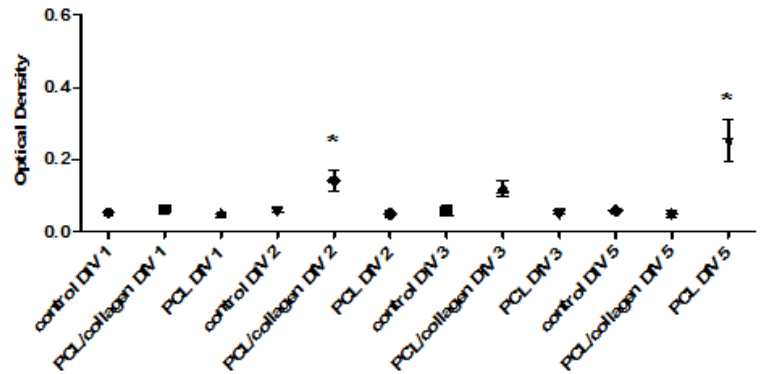

Fig. 3. Proliferation of hWJMSCs on different nanofiber materials over five days in vitro.

\section{Discussion}

Results indicate that the PCL and PCL/collagen scaffolds had similar morphological and structural characteristics (fiber diameter showed no significant differences); however, the mechanical properties were significantly different. This is because the electrospinning process was optimized for each type of solution to create scaffolds of similar fiber diameter. PCL/collagen scaffolds produced a significant increase in proliferation of hWJMSCs relative to PCL scaffolds alone, suggesting an ideal potential for the combined use of PCL/collagen scaffold and hWJMSCs for tissue repair/regeneration applications.

\section{ACKNOWLEDGMENTS}

We would like to acknowledge USDA National Center for Agriculture Utilization Research in Peoria, IL for utilization of the Instron machine for tensile testing, and Dr. Wei Du, Director of the Imaging Center at SIU School of Medicine, Springfield, IL, for assistance with SEM imaging.

\section{REFERENCES}

[1] Pham, Q, Sharma, U, \& Mikos, A. (2006). "Electrospinning of Polymeric Nanofibers for Tissue Engineering Applications: A Review," Tissue Engineering, Vol. 12, No. 5, pp. 1197-1211.

[2] Venugopal, J, Zhang, Y, \& Ramakrishna, S. (2006). "In Vitro Culture of Human Dermal Fibroblasts on Electrospun Polycaprolactone Collagen Nanofibrous Membrane," Artificial Organs, Vol. 30, No. 6, pp. 440446. 